\title{
ORIGINAL ARTICLE The origin of circulating CD36 in type 2 diabetes
}

\author{
MJ Alkhatatbeh ${ }^{1,2}$, AK Enjeti ${ }^{2,3}$, S Acharya ${ }^{4}$, RF Thorne ${ }^{1,2,5}$ and LF Lincz ${ }^{2,3,5}$
}

OBJECTIVE: Elevated plasma levels of the fatty acid transporter, CD36, have been shown to constitute a novel biomarker for type 2 diabetes mellitus (T2DM). We recently reported such circulating CD36 to be entirely associated with cellular microparticles (MPs) and aim here to determine the absolute levels and cellular origin(s) of these CD36 + MPs in persons with T2DM.

DESIGN: An ex vivo case-control study was conducted using plasma samples from 33 obese individuals with T2DM (body mass index $(\mathrm{BMI})=39.9 \pm 6.4 \mathrm{~kg} \mathrm{~m}^{-2}$; age $=57 \pm 9$ years; 18 male: 15 female) and age- and gender-matched lean and obese non-T2DM controls $\left(\mathrm{BMI}=23.6 \pm 1.8 \mathrm{~kg} \mathrm{~m}^{-2}\right.$ and $33.5 \pm 5.9 \mathrm{~kg} \mathrm{~m}^{-2}$, respectively). Flow cytometry was used to analyse surface expression of CD36 together with tissue-specific markers: CD41, CD235a, CD14, CD105 and phosphatidyl serine on plasma MPs. An enzyme-linked immunosorbent assay was used to quantify absolute CD36 protein concentrations.

RESULTS: CD36 + MP levels were significantly higher in obese people with T2DM $(P<0.00001)$ and were primarily derived from erythrocytes (CD235a $+=35.8 \pm 14.6 \%)$; although this did not correlate with haemoglobin $A_{1 c}$. By contrast, the main source of CD36 + MPs in non-T2DM individuals was endothelial cells (CD105 $+=40.9 \pm 8.3 \%$ and $33.9 \pm 8.3 \%$ for lean and obese controls, respectively). Across the entire cohort, plasma CD36 protein concentration varied from undetectable to $22.9 \mu \mathrm{g} \mathrm{ml}^{-1}$ and was positively correlated with CD36 + MPs measured by flow cytometry $(P=0.0006)$ but only weakly associated with the distribution of controls and T2DM $(P=0.021)$. Multivariate analysis confirmed that plasma CD36 + MP levels were a much better biomarker for diabetes than CD36 protein concentration ( $P=0.009$ vs $P=0.398$, respectively).

CONCLUSIONS: Both the levels and cellular profile of CD36 + MPs differ in T2DM compared with controls, suggesting that these specific vesicles could represent distinct biological vectors contributing to the pathology of the disease.

Nutrition and Diabetes (2013) 3, e59; doi:10.1038/nutd.2013.1; published online 4 February 2013

Keywords: type 2 diabetes mellitus; microparticles; scavenger receptor; soluble CD36

\section{INTRODUCTION}

CD36 is a multifunctional signalling molecule with several known ligands, including thrombospondin-1, long chain fatty acids and both native and atherogenic lipoproteins, including oxidised lowdensity and high-density lipoproteins (reviewed in Thorne et al. ${ }^{1}$ ). Expressed on a variety of cells and tissues, including platelets, erythrocytes, monocytes, endothelial cells and leucocytes, ${ }^{2}$ CD36 is intimately involved in lipid metabolism and homoeostasis and has been strongly implicated in pathological conditions associated with metabolic dysregulation, including obesity, insulin resistance, diabetes, diabetic nephropathy and atherosclerosis., ${ }^{3,4}$ More recently, a circulating form of CD36, termed soluble (s) CD36, was identified in human plasma as a novel biomarker for type 2 diabetes mellitus (T2DM). ${ }^{5}$ Further analysis showed that sCD36 levels clustered with markers of insulin resistance and elevated SCD36 correlated with increased risk of T2DM. ${ }^{6}$ These authors also found that sCD36 could be a marker of atherosclerotic plaque instability, a major determinant in the risk of acute coronary syndromes. ${ }^{7}$ In addition, sCD36 was elevated in prediabetic conditions, including polycystic ovary syndrome ${ }^{8}$ and was identified as a novel marker of liver injury in patients with altered glucose metabolism. ${ }^{9}$ Even in a non-diabetic healthy population, plasma sCD36 was significantly associated with markers of insulin resistance, atherosclerosis and fatty liver. ${ }^{10}$ All of these studies used an enzyme-linked immunosorbent assay (ELISA) for CD36 using relative arbitrary units. Despite its apparent significance, normal absolute ranges for plasma CD36 protein concentration have not been quantified, and the cellular source(s) of plasma CD36 has not been identified.

The CD36 glycoprotein is known to be profoundly resistant to proteolytic cleavage, and this property is thought to result from its heavily $\mathrm{N}$-glycosylated extracellular domain. ${ }^{11}$ Consequently, we hypothesised that circulating CD36 was unlikely to constitute a cleaved cell-free variant of the receptor. Studies from our laboratory confirmed this as sCD36 in plasma was shown to be entirely associated with circulating microparticles (MPs). ${ }^{12}$ Corroborating our findings, a report using mass spectrometry confirmed that CD36 was one of 130 'core' proteins of plasma MPs in a cohort of patients with a high rate of cardiovascular disease (86\%) and diabetes (75\%). ${ }^{13}$

MPs are small $(0.1-1 \mu \mathrm{m}$ in diameter) membranous microvesicles that can be specifically and selectively released from virtually any eukaryotic cell. They retain many features of their cell of origin, so that examination of their antigen expression can reveal their cellular source. ${ }^{14}$ Greater than $90 \%$ of circulating MPs are thought to be platelet derived, ${ }^{15}$ present in the plasma of normal healthy individuals at an estimated concentration of up to $50 \mu \mathrm{g} \mathrm{ml}^{-1}$. ${ }^{16}$ However, their numbers and cellular source can

\footnotetext{
${ }^{1}$ Cancer Research Unit, School of Biomedical Sciences and Pharmacy, Faculty of Health, the University of Newcastle, Newcastle, NSW, Australia; ${ }^{2}$ Hunter Medical Research Institute, New Lambton, NSW, Australia; ${ }^{3}$ Hunter Haematology Research Group, Calvary Mater Newcastle Hospital, Waratah, NSW, Australia and ${ }^{4}$ Department of Diabetes, John Hunter Hospital, Hunter New England Local Health District, New Lambton, NSW, Australia. Correspondence: Dr LF Lincz, Hunter Haematology Research Group, Calvary Mater Newcastle Hospital, Waratah, NSW 2298, Australia.
}

E-mail: lisa.lincz@newcastle.edu.au

${ }^{5}$ These authors contributed equally to the study.

Received 24 September 2012; revised 3 December 2012; accepted 24 December 2012 
change dramatically in various pathological states, ${ }^{17}$ including T2DM and cardiovascular disease. ${ }^{18}$ Importantly, MPs are enriched with bioactive proteins and nucleic acids, and there is growing evidence that they form a network of biological vectors able to transfer cellular components to remote areas of the body in order to mediate specific changes in their target cells. ${ }^{19}$ Thus, rather than being a simple biomarker, CD36 may, in fact, identify a specific subset of MPs that act as mediators in the pathogenesis of metabolic disease.

The aim of the present study was to quantify the absolute plasma levels of CD36 protein concentration and CD36+MPs in healthy and diabetic individuals, to discover their cellular source and ultimately determine whether any differences exist in these parameters relative to obesity and/or T2DM.

\section{SUBJECTS AND METHODS}

\section{Subjects}

Cases consisted of a sub-population $(n=33)$ randomly selected from a cohort of 120 patients with poorly controlled T2DM recruited from the John Hunter Hospital Diabetes Clinic, Hunter New England Area Health Service, NSW, Australia. The median duration (interquartile range) of disease was $10.0(5.0-17.0)$ years. Further information about the cases is provided in Supplementary Table S1. These were age- and gendermatched to plasma samples from 33 lean (body mass index (BMI) $20-25 \mathrm{~kg} \mathrm{~m}^{-2}$ ) and 33 obese (BMI $>25 \mathrm{~kg} \mathrm{~m}^{-2}$ ) healthy controls sourced from previously collected plasma samples from 400 healthy Red Cross blood donors. The study was approved by the University of Newcastle and Hunter New England Area Human Research Ethics Committees, and informed consent was obtained from all the participants. Peripheral blood samples were collected into 3.2\% Na citrate Vacuette containers (Greiner Bio-One $\mathrm{GmbH}$, Frickenhausen, Germany) and platelet-free plasma was prepared by serial double centrifugation $(15 \mathrm{~min}$ at $2100 \mathrm{~g})$ within $2 \mathrm{~h}$ of collection. Aliquoted samples were stored at $-80^{\circ} \mathrm{C}$, then thawed at $37^{\circ} \mathrm{C}$ immediately before analysis.

\section{Analysis of MPs by flow cytometry}

Staining and analysis of MPs was performed essentially as previously described ${ }^{12,20}$ and according to guidelines established by the International Society on Thrombosis and Haemostasis Vascular Biology SSC on the Standardisation of FMC-based PMP enumeration by flow cytometry incorporating modifications suggested for the BD FACS Canto (BD Biosciences, San Jose, CA, USA). ${ }^{21}$ To calibrate the cytometer, a blend of 2:1:1 0.5-, 0.9- and 3- $\mu \mathrm{m}$ diameter fluorescent beads (Megamix, Biocytex, Marseille, France) was used to ensure adequate FSC resolution and set the lower MPs' detection limit according to the manufacturer's instructions. A $10-\mu \mathrm{l}$ aliquot of platelet-free plasma was incubated at room temperature for $30 \mathrm{~min}$ with an antibody against CD36 (clone 11H5) conjugated to DyLight-488 in various combinations with CD41-phycoerythrin (CD41-PE; clone PL2-49, Biocytex; platelet marker), CD14-PE (clone TÜK4, Miltenyi Biotec, Teterow, Germany; monocyte marker), CD235a-allophycocyanin (CD235a-APC; clone GA-R2 (HIR2), BD Pharmingen, San Diego, CA, USA; erythrocyte marker), CD105-PE (clone 1G2, Beckman Coulter, Brea, CA USA; endothelial marker) and Annexin V-APC (eBioscience, San Diego, CA, USA; to measure phosphatidylserine (PS) exposure as a marker of vesicles derived from activated or apoptotic cells). All assays were diluted in phosphate-buffered saline (PBS; without $\mathrm{Ca}^{+2}$ and $\mathrm{Mg}^{+2}$ ), with the exception of those containing Annexin V, which were diluted in calciumrich binding buffer as supplied by the manufacturer (eBioscience). TruCount counting tubes (BD Biosciences) were used to enable absolute MP quantification, and events were collected for $60 \mathrm{~s}$ at low flow rate on a BD FACS Canto (BD Biosciences) before analysis using FACS Diva software (BD Biosciences). The absolute number of MPs in each plasma sample was calculated using the formula: MPs $\mu l^{-1}=(\mathrm{MP}$ count/bead count $) \times$ (total beads in Trucount tube/test volume). The reproducibility of the assay was determined by staining and analysing a series of five different plasma samples in triplicate on five separate occasions. The intra- and interassay variability was thus calculated to be $11.3 \%$ and $10.6 \%$, respectively.

\section{CD36 ELISA}

ELISA plates (Microlon high-binding; Greiner Bio One, West Heidelberg Australia) were precoated with anti-CD36 mAb $(11 \mathrm{H} 5 ; 500 \mathrm{ng}$ per well) overnight at $4{ }^{\circ} \mathrm{C}$ before blocking with $5 \%(\mathrm{w} / \mathrm{v})$ skimmed milk powder in PBS buffer containing $0.05 \%$ (v/v) Tween-20. Serial dilutions of platelet purified $C D 36^{22}$ were prepared in PBS for the standard curve, and plasma samples were diluted 1/4 in PBS before being applied to coated wells for $2 \mathrm{~h}$ at room temperature. Following a further $2 \mathrm{~h}$ incubation with a rabbit polyclonal antibody against CD36, immunocomplexes were detected with anti-rabbit lgG-horseradish peroxidise conjugate (1:5000 (v/v); Bio-Rad, Richmond, CA, USA) and developed with SIGMAFAST OPD (O-phenylenediamine dihydrochloride, Sigma-Aldrich, Castle Hill, NSW, Australia). Reactions were stopped after $30 \mathrm{~min}$ by addition of $3 \mathrm{M} \mathrm{HCL}$, and absorbance was measured at $490 \mathrm{~nm}$ using a SpectraMAX microplate reader (Molecular Devices, Hawthorne East, Australia). All washes were performed with PBS-Tween-20 buffer and all incubations were at room temperature unless otherwise indicated.

The average background absorbance was subtracted from the mean of each duplicate test. Sample concentration was determined from a standard curve constructed by linear regression after plotting concentration vs absorbance on logarithmic scales. The minimum level of CD36 protein detection was $78 \mathrm{ng} \mathrm{ml}^{-1}$. The reproducibility of the ELISA was ensured by repeating a subset of samples on different days. The mean intra- and interassay coefficients of variation from these experiments were $14.1 \%$ and $9.5 \%$, respectively.

\section{Statistical analysis}

Data for continuous variables were expressed as mean \pm s.d. or median (interquartile range) where appropriate. Variables that were not normally distributed were either analysed using non-parametric tests or log transformed before analysis as indicated. Differences in mean levels of normally distributed continuous variables were assessed using the Student's $t$-test, while the non-parametric Mann-Whitney $U$ test was used to detect differences between medians. Correlations between continuous variables were assessed by Pearson Product-Moment, while their association with quartiles of plasma CD36 protein concentration was determined by ordinal multinomial logistic regression. Frequencies of dichotomous variables were evaluated using Fisher's exact test, and multivariate analysis was performed by unconditional multiple logistic regression. All calculations were performed with Statistica v10.0 (StatSoft, Tulsa, OK, USA) using two-tailed tests, and $P$-values $<0.05$ were considered statistically significant.

\section{RESULTS}

\section{CD36 + MPs are elevated in T2DM}

General characteristics of the study group are provided in Table 1. The cohorts were well matched in terms of gender and age, and differed mainly in terms of BMI. In this regard, the diabetic participants were also significantly more obese than the obese controls $(P<0.001)$. This was reflected by differences in white blood cell count parameters, which are known to be positively associated with obesity and also weakly influenced by plasma insulin levels. ${ }^{23,24}$

Flow cytometry was used to detect expression of CD36 as well as other specific cellular markers on circulating MPs in plasma samples collected from lean and obese controls together with obese diabetics. As shown in Figure 1, a substantial number of MPs in plasma from all the cohorts were found to express CD36, with levels corresponding to approximately 50\% of those of platelet-derived MPs. Importantly, CD36 + MPs were significantly higher in the obese T2DM cohort (median (interquartile range) per $\mu \mathrm{l}$ of plasma $=1063(785-1276))$ compared with the obese controls (415 (295-5020), $P<0.00001)$. Similar results were observed for MPs expressing markers specific to platelets (CD41), erythrocytes (CD235a) and monocytes (CD14), as well as the general apoptotic/activation marker PS (as detected by Annexin V). The proportion of platelet-derived MPs that expressed PS was also greatly increased in the diabetic cohort. Only the number of MPs expressing the endothelial marker (CD105) was found to be similar between all the three cohorts. There was no significant difference in any of the MP subsets between lean and obese controls.

Correlation analysis of the combined cohorts revealed that the number of MPs expressing CD41, CD235a and CD14 were not 
Table 1. Demographics and blood parameters of study participants

\begin{tabular}{|c|c|c|c|}
\hline Demographics & Lean controls & Obese controls & Obese T2DM \\
\hline Number $(n)$ & 33 & 33 & 33 \\
\hline Gender (M/F) & $18 / 15$ & $18 / 15$ & $18 / 15$ \\
\hline Age (years) & $55.7 \pm 7.1$ & $56.2 \pm 8.1$ & $57.1 \pm 8.8$ \\
\hline Height $(\mathrm{cm})$ & $170.2 \pm 8.0$ & $171.4 \pm 9.7$ & $168.2 \pm 10.1$ \\
\hline Weight (kg) & $68.6 \pm 9.0$ & $98.4 \pm 17.5^{+++}$ & $110.5 \pm 20.2^{*}$ \\
\hline BMI $\left(\mathrm{kg} \mathrm{m}^{-2}\right)$ & $23.6 \pm 1.8$ & $33.6 \pm 6.0^{+++}$ & $40.0 \pm 6.4^{* * *}$ \\
\hline Current smokers & 1 & 1 & 3 \\
\hline \multicolumn{4}{|c|}{ Blood counts $\left(\times 10^{9} \mathrm{I}^{-1}\right)$} \\
\hline Leucocytes & $6.22 \pm 1.79$ & $6.84 \pm 1.19$ & $8.32 \pm 2.47^{* *}$ \\
\hline Neutrophils & $3.22(2.78-4.33)$ & $4.11(3.44-4.88)$ & $4.33(3.78-5.89)^{*}$ \\
\hline Lymphocytes & $1.74 \pm 0.43$ & $1.96 \pm 0.50^{+}$ & $2.40 \pm 1.13^{*}$ \\
\hline Monocytes & $0.48 \pm 0.15$ & $0.52 \pm 0.12$ & $0.62 \pm 0.21^{*}$ \\
\hline Eosinophils & $0.17 \pm 0.09$ & $0.19 \pm 0.11$ & $0.19 \pm 0.11$ \\
\hline Erythrocytes & $4.66 \pm 0.39$ & $4.76 \pm 0.45$ & $4.55 \pm 0.39^{*}$ \\
\hline Platelets & $215.15 \pm 34.40$ & $238.15 \pm 52.80^{+}$ & $224.21 \pm 63.08$ \\
\hline Haemoglobin $\left(\mathrm{gl}^{-1}\right)$ & $140.50 \pm 10.87$ & $142.09 \pm 14.89$ & $137.58 \pm 13.07$ \\
\hline
\end{tabular}

Abbreviations: BMI, body mass index; $\mathrm{F}$, female; M, male; T2DM, type 2 diabetes mellitus. ${ }^{+} P<0.05,{ }^{+++} P<0.001$ vs lean controls; ${ }^{*} P<0.05,{ }^{* *} P<0.01$, ${ }^{* * *} P<0.001$ vs obese controls. Data expressed as mean \pm s.d. or median (interquartile range) and log transformed before statistical analysis as appropriate.

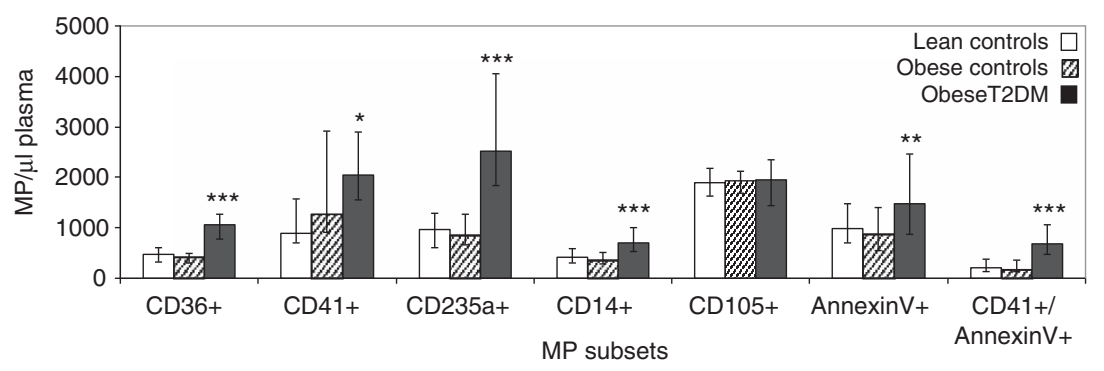

Figure 1. Levels of MP subsets in lean controls, obese controls and obese T2DM individuals. Data expressed as medians with bars indicating interquartile ranges. ${ }^{*} P<0.05,{ }^{* *} P<0.001,{ }^{* * *} P<0.00001$ vs obese controls.

related to the peripheral blood count of their respective cells of origin (that is, platelets, $R=0.19, P=0.073$; erythrocytes, $R=-0.03, P=0.727$; monocytes $R=0.19, P=0.054$ ); however, there was a strong positive correlation between the total leucocyte count and most MP subsets (CD36+MP, $R=0.30$, $P=0.003 ; \quad \mathrm{CD} 41+\mathrm{MP}, \quad R=0.41, \quad P<0.001 ; \quad \mathrm{CD} 235 \mathrm{a}+\mathrm{MP}$, $R=0.34, P=0.001 ; \mathrm{CD} 14+\mathrm{MP}, R=0.16 P=0.113 ; \mathrm{CD} 105+\mathrm{MP}$, $R=0.17, \quad P=0.092$; Annexin $\mathrm{V}+\mathrm{MP}, \quad R=0.18, \quad P=0.08$; and $\mathrm{CD} 41+/$ Annexin $\mathrm{V}+\mathrm{MP}, \quad R=0.25, \quad P=0.011)$. None of the peripheral blood count parameters or MP levels were related to participants' age (data not shown).

CD36 + MPs in T2DM are mainly derived from erythrocytes

During the course of multicolour flow cytometry analysis for individual MP subsets, it became clear that the majority of circulating CD36+MPs co-expressed other markers being investigated. In an effort to determine the primary origin of these CD36 + MPs, the percentage expressing each of the analysed markers was calculated. As illustrated in Figure 2, the primary source of $\mathrm{CD} 36+\mathrm{MPs}$ in obese T2DM individuals was from erythrocytes $(35.8 \pm 14.6 \%)$, occurring at levels that were significantly greater than in obese controls $(22.2 \pm 9.0 \% ; P<0.00001)$. By contrast, the main source of CD36+MPs in non-diabetic individuals was endothelial cells $(40.9 \pm 8.3 \%$ and $33.9 \pm 8.3 \%$ for lean and obese controls, respectively) followed closely by platelets $(16.8 \pm 8.3 \%$ and $23.8 \pm 12.0 \%)$. Overall, only the subset of CD36+ MPs expressing PS was specifically increased in diabetes, while an increase in the percentage of CD36 + MPs derived from platelets was specific to obesity and an increase in erythrocyte-derived
CD36 + MPs was common to both conditions, albeit highest in diabetes.

There was no correlation between levels of any of the CD36+ MP subsets and any of the peripheral blood parameters listed in Table 1, with the exception of the percentage of CD41 + /CD36 + MPs, which was weakly associated with platelet counts $(R=0.22, P=0.030)$.

Erythrocyte-derived MPs in T2DM do not correlate with haemoglobin $\mathrm{A}_{1 \mathrm{c}}\left(\mathrm{HbA}_{1 \mathrm{c}}\right)$

We speculated that high levels of glycated haemoglobin (as measured by plasma $\mathrm{HbA}_{1 \mathrm{c}}$ ) within diabetic erythrocytes may trigger increased apoptosis and/or shedding of this cellular toxin in membrane-bound vesicles. The diabetic cohort was known to have poorly controlled diabetes, with average $\mathrm{HbA}_{1 \mathrm{c}}$ levels of $7.7 \pm 1.1 \%$. However, there was no correlation between these values and the overall number of CD235a + MPs or the percentage of $\mathrm{CD} 36+\mathrm{MPs}$ expressing this marker (Figure 3 ). This was confirmed using an extended cohort $(n=44)$ of obese people with T2DM (Supplementary Figure S1).

Plasma CD36 + MPs are a better biomarker for T2DM than protein concentration

An ELISA assay was devised to measure the absolute levels of CD36 protein in the same plasma samples used in the above experiments. These levels were determined to range from undetectable in $31 / 99$ participants to as high as $22.9 \mu \mathrm{g} \mathrm{ml}^{-1}$. As shown in Figure 4 , the median levels progressively increased from lean to obese controls to obese diabetic individuals (1.71 vs 


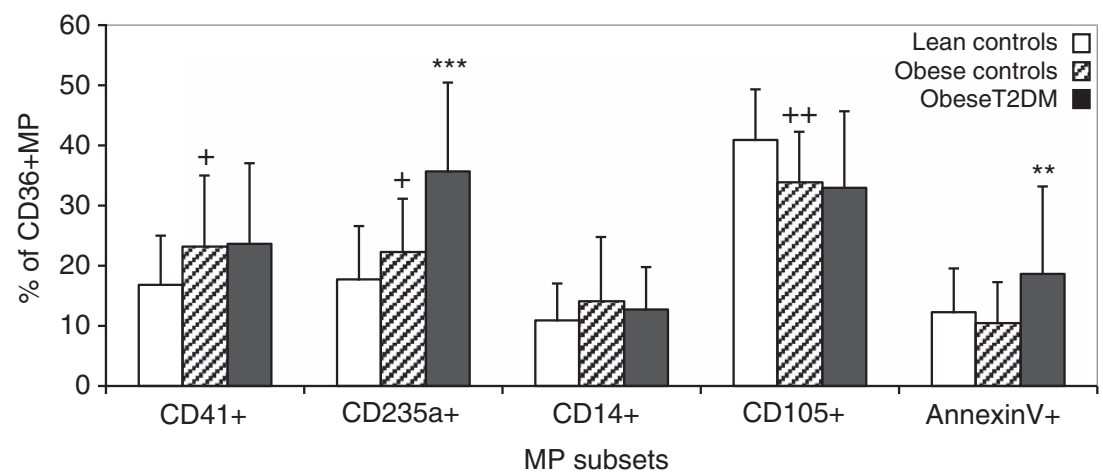

Figure 2. Percentage of $C D 36+M P$ derived from specific MP subsets in lean controls, obese controls and obese T2DM individuals. Data expressed as mean and s.ds. ${ }^{+} P<0.05,{ }^{+}+P<0.01$ vs lean controls; ${ }^{* *} P<0.001,{ }^{* * *} P<0.00001$ vs obese controls.
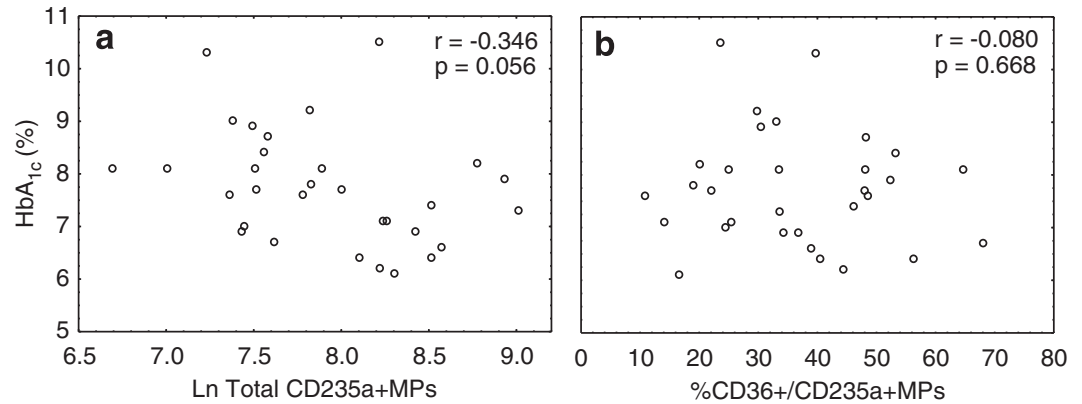

Figure 3. Scatter plots of $\mathrm{HbA}_{1 c}$ plasma concentrations vs erythrocyte-derived MPs in obese T2DM individuals. (a) Ln Total CD235a + MPs; (b) percentage of CD36 + /CD235a + MPs.

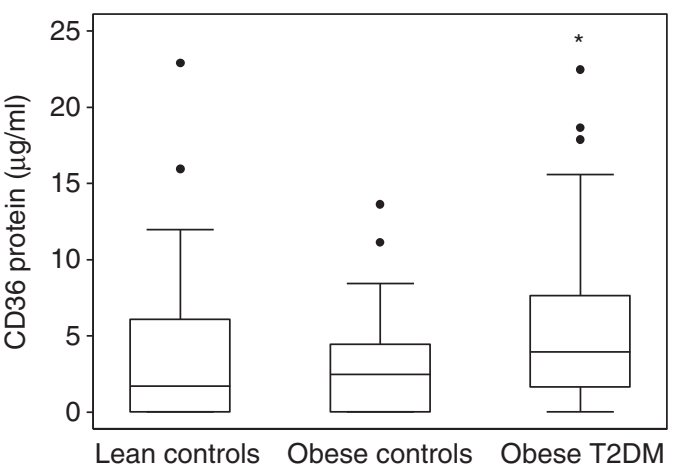

Figure 4. Plasma levels of CD36 protein measured by ELISA in lean controls, obese controls and obese T2DM individuals. Data expressed as median, interquartile range (boxes), upper and lower adjacent values (whiskers) and individual outliers (circles). ${ }^{*} P<0.05$ compared with obese controls.

2.47 vs $3.90 \mu \mathrm{g} \mathrm{ml}^{-1}$ ), with the difference between the latter two cohorts being statistically significant $(P=0.036)$. In order to accurately assess the relationship between protein and MP levels of $C D 36$, the CD36 protein concentrations were log transformed and divided into quartiles to accommodate the large number of data points below the limit of detection for the assay. This was then compared with the normalised CD36 + MP levels for each lean, obese and obese diabetic participant, with the results illustrated in Figure 5. When all cohorts were combined, it became clear that there was a strong correlation between CD36+MP levels and CD36 protein concentrations $(P=0.0006)$. However, the individual distributions of lean, obese and obese diabetic participants was only weakly correlated with quartiles of plasma

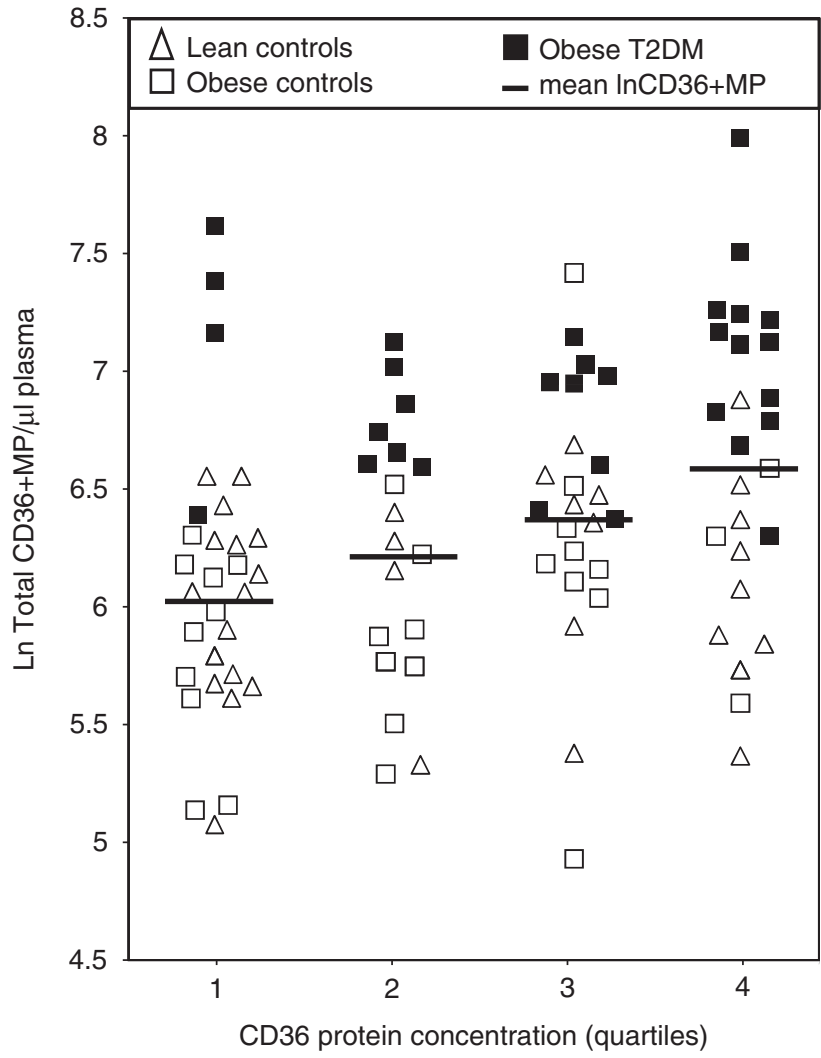

Figure 5. Quartiles of plasma CD36 protein concentration measured by ELISA compared with numbers of circulating CD36+MPs measured by flow cytometry in lean controls, obese controls and obese T2DM individuals. 
CD36 protein concentration $(P=0.021)$, with diabetic patients appearing in all the quartiles. By contrast, none of the diabetic patients had CD36 + MP levels in the lower half of the y axis as illustrated on the graph. Multivariate analysis confirmed that even when corrected for BMI and blood parameters, CD36 + MP levels were a much better marker of diabetes than CD36 protein levels $(P=0.009$ vs $P=0.398$, respectively). However, neither parameter was significantly associated with obesity in the control cohorts $(P=0.173$ for $\mathrm{CD} 36+\mathrm{MP}$ and $P=0.906$ for quartiles of CD36 protein levels).

\section{DISCUSSION}

This study represents the first thorough investigation of the origin of circulating CD36 in human plasma. The results confirm our initial findings, which concluded that the proposed soluble form of CD36 (sCD36) $)^{5}$ is not truly soluble but entirely associated with plasma MPs. ${ }^{12}$ In the present study, we have gone on to further demonstrate that these $\mathrm{CD} 36+$ MPs are readily detectable in both T2DM and non-T2DM individuals, occurring at levels corresponding to approximately half that of platelet MPs. Importantly, the cellular source for the majority of CD36+MPs could be accounted for using markers specific to platelet, endothelial and erythrocyte antigens. However, the relative amounts of each source differed between diabetic individuals and controls, with $\mathrm{CD} 36+\mathrm{MPs}$ being mainly derived from erythrocytes in T2DM as compared with endothelial cells in healthy controls.

There have been many reports of increased levels of circulating MPs in various pathological states associated with chronic inflammation and hypercoagulation, including obesity, ${ }^{25}$ the metabolic syndrome, ${ }^{26}$ and T2DM. ${ }^{27}$ Indeed, MPs are well known to exhibit both pro-coagulant and pro-inflammatory properties. ${ }^{17}$ Thus, the association of high MP counts with increased leucocyte count, a very basic but widely recognised indication of inflammatory conditions, is not unexpected. The most commonly examined MPs are those derived from platelets and endothelial cells and often incorporate various activation markers; ${ }^{28}$ while others target monocyte-derived $\mathrm{MPs}^{29}$ and some focus on expression of molecules that may be specific to a particular function, such as Tissue Factor for its role in atherogenesis. ${ }^{30}$ Regardless of the MP species, levels are nearly always found to be increased in T2DM compared with controls. Our results are consistent with this trend, showing significantly higher levels of MPs expressing PS, CD41, CD235a, CD14 and CD36 in obese T2DM compared with obese controls. Thus, the absolute increase of CD36 + MP levels could have simply been due to an overall increase in MP production associated with inflammation. However, analysis of specific subsets of CD36 + MPs revealed that these were not universally increased but rather particularly derived from erythrocytes.

Very few investigations of circulating MPs have considered erythrocyte-derived MPs in any disease context. Most of the interest has occurred with respect to blood transfusion and the mechanisms of erythrocyte aging. Throughout their short existence, erythrocytes gradually lose $20 \%$ of their haemoglobin and corresponding surface area through the shedding of vesicles ${ }^{31}$ in a vital mechanism that is postulated to rid the erythrocyte of damaged membrane in order to prolong its lifespan. ${ }^{32}$ As a consequence, the favourable surface area-tovolume ratio decreases, rendering old erythrocytes less deformable. In support of increased erythrocyte MP release in diabetes, a recent report has shown that erythrocyte deformability is impaired in patients with coronary artery disease coupled with diabetes, and this was inversely correlated to both glucose concentration and $\mathrm{HbA}_{1 \mathrm{c}}{ }^{33}$ Furthermore, analysis of MPs in stored erythrocyte transfusion bags has revealed that these vesicles are selectively enriched with the major modified haemoglobin species, including $\mathrm{HbA}_{1 \mathrm{c}}{ }^{34}$ Thus, it would logically follow that high plasma glucose levels in poorly controlled diabetes that lead to increased percentage of $\mathrm{HbA}_{1 \mathrm{c}}$ would result in increased shedding of MPs from erythrocytes. It was surprising then that we found no correlation between individual $\mathrm{HbA}_{1 c}$ and circulating erythrocyte-derived MP levels in the our diabetic cohort. This unexpected finding clearly warrants further investigation, but nevertheless, there is some evidence to associate the level of diabetes control and the increased erythrocyte MPs in diabetes.

Outside of the current work we know of only two small studies that have previously considered erythrocyte-derived MPs in T2DM, each giving rise to different conclusions. Agouni et al. ${ }^{26}$ found that erythrocyte-derived MPs were significantly increased in a cohort of 43 patients with metabolic syndrome, a third of whom also had diabetes. By contrast, Diamant et $a l^{30}$ showed no difference between CD235a + MPs in 18 controls and 16 patients with normal BMI and uncomplicated diabetes of short duration. The differences may lie within the cohorts themselves as diabetic subjects analysed by Agouni et al. ${ }^{26}$ had relatively high $\mathrm{HbA}_{1 c}$ levels compared with those employed by Diamant et al. ${ }^{30}$ $(6.3 \pm 0.13$ (ref. 26), vs $5.8 \pm 1.2$ (ref. 30), respectively, compared with the normal range of $3.5-5.5 \%)$. In the present study, the $\mathrm{HbA}_{1 \mathrm{c}}$ levels were even higher $(7.7 \pm 1.1 \%$ in obese diabetic individuals), thereby providing a rationale to reconcile erythrocyte MPs levels with the extent of diabetes control.

Levels of circulating endothelial cell MPs are believed to reflect cellular damage and are increasingly being used as markers of endothelial cell dysfunction in various conditions, such as cardiovascular disease. ${ }^{35}$ However, we found no difference in overall endothelial cell-derived MPs between T2DM and controls, consistent with independent reports by Sabatier et al., ${ }^{36}$ and Tsimerman et al. ${ }^{37}$ This is in contrast to a report of increased total endothelial $(C D 144+)$ MPs in the metabolic syndrome, ${ }^{38}$ and a case control study by Tramontano et $a l^{28}$ who found $\mathrm{CD} 105+\mathrm{MP}$ levels to be the only independent predictor of diabetes. These conflicting reports may simply reflect the diabetic cohort being analysed, as endothelial MP profiles have been shown to vary specifically with accompanying diabetic vascular complications, ${ }^{39}$ which were not considered in the present study. Alternatively, medications consumed by the cohort to minimise vascular complications could also affect endothelial MP levels, as suggested by the findings that the latter are reduced by pioglitazone therapy in patients with the metabolic syndrome ${ }^{40}$ but increased in response to atorvastatin (statins were prescribed to over $60 \%$ of our T2DM cohort as indicated in Supplementary Table S1) in patients with peripheral arterial occlusive disease. ${ }^{41}$ Independent of these considerations, we found that levels of endothelial MPs measured by detection of CD105 were unusually high; even the control cohorts had levels that surpassed that of platelet MPs. Because CD105 is not specific for endothelial cells (it can also be expressed on activated macrophages, fibroblasts and smooth muscle cells), we have omitted any CD105+MPs that co-expressed the leucocyte-specific marker CD45; however, this was negligible. Others have observed endothelial MP levels of similar enormity in controls using antibodies to comparable non-specific markers such as CD31 (found on platelets, monocytes, granulocytes, and B-cells), even when combined with CD42 to exclude platelets. ${ }^{42}$ Alternatively, particular species of endothelial MPs have been shown to increase after prolonged storage at $-80^{\circ} \mathrm{C} .{ }^{43}$ The controls for the present study were collected before the cases and thus may have endothelial MP levels that are artefactually increased due to longer storage times. Although all of the samples were processed in identical fashion, there is no consensus on the effects of long-term storage for MP enumeration, and this may also explain why the 
control endothelial MP levels appeared to be equal to that of the T2DM patients.

This is the first study to quantify CD36 protein levels in human plasma. These were found to vary widely from undetectable $\left(<78 \mathrm{ng} \mathrm{ml}^{-1}\right)$ to as high as $22.9 \mu \mathrm{g} \mathrm{ml}^{-1}$. These values are relatively high compared with other plasma proteins such as SCD40L and sICAM (soluble intercellular adhesion molecule), which are in the order of 3 and $300 \mathrm{ng} \mathrm{ml}^{-1}$, respectively. The only other published study to use a quantitative CD36 ELISA was performed by Chmielewski et al., ${ }^{44}$ but their assay differed from ours in that they used serum rather than plasma samples. Although serum has been reported to contain 10 times more platelet MPs than plasma, ${ }^{45}$ it is not clear how much CD36 is contributed by these vesicles that have been produced by artificial platelet activation in vitro. In addition, other CD36-expressing MPs present before activation may become entangled in the clot that forms. Chmielewski et al. found the median CD36 protein concentration in serum to be a low $25.3 \mathrm{ng} \mathrm{ml}^{-1}$ in non-diabetic patients, suggesting that the latter may be true. The recently released commercial ELISA kits for quantification of CD36 in human plasma have a reported sensitivity of $1.0 \mathrm{ng} \mathrm{ml}^{-1}$ (Adipo Bioscience, Santa Clara, CA, USA) and should facilitate further studies in this area.

The high variability in plasma CD36 protein concentrations detected by ELISA may reflect actual individual differences in cellular expression of CD36. Protein levels on the surface of monocytes and platelets are largely determined by the inheritance of common CD36 gene polymorphisms, ${ }^{46,47}$ but this expression is also subject to further exogenous modulation. Of particular relevance to the present cohort, statins have been shown to decrease CD36 expression on monocytes and have been associated with lower levels of sCD36 in diabetic individuals. ${ }^{44,48}$ Conversely, CD36 expression can be increased by both fat in the diet $^{49}$ and hyperglycaemia. ${ }^{50}$ If the number of CD36 molecules on MPs is reflective of that of the parent cell, then any analysis of absolute circulating CD36 protein levels will be subject to these various confounders. By contrast, quantification of the number of MPs expressing CD36 will be less compromised by individual protein expression levels. For these reasons, it is perhaps not surprising that plasma CD36 + MP levels were a much better marker of T2DM than CD36 protein concentration per se.

\section{CONCLUSIONS}

This is the first study to establish the source of the putative diabetic marker, plasma CD36 (CD36 + MPs), in T2DM. Compared with plasma CD36 protein concentration, CD36 + MPs were found to be a better independent marker of T2DM with the advantage of facilitated measurement by multicolour flow cytometry. The increased percentage of CD36 + MPs originating from erythrocytes in T2DM merits further investigation into the mechanism underlying this pathophysiology. Future directions will focus on determining whether these specific vesicles have a particular role in contributing to the pathology of this disease.

\section{CONFLICT OF INTEREST}

The authors declare no conflict of interest.

\section{ACKNOWLEDGEMENTS}

This study was supported by a University of Newcastle Strategic Pilot Grant and a Hunter Medical Research Institute (HMRI) Special Project Grant, supported by Lions District 201 N3 Diabetes Foundation, awarded to RFT and LFL. MJA is supported by a scholarship from the Jordan University of Science and Technology (JUST).

\section{REFERENCES}

1 Thorne RF, Mhaidat NM, Ralston KJ, Burns GF. CD36 is a receptor for oxidized high density lipoprotein: implications for the development of atherosclerosis. FEBS Lett 2007; 581: 1227-1232.

2 Silverstein RL. Inflammation, atherosclerosis, and arterial thrombosis: role of the scavenger receptor CD36. Cleve Clin J Med 2009; 76(Suppl 2): S27-S30.

3 Febbraio M, Hajjar DP, Silverstein RL. CD36: a class B scavenger receptor involved in angiogenesis, atherosclerosis, inflammation, and lipid metabolism. J Clin Invest 2001; 108: 785-791.

4 Susztak K, Ciccone E, McCue P, Sharma K, Bottinger EP. Multiple metabolic hits converge on CD36 as novel mediator of tubular epithelial apoptosis in diabetic nephropathy. PLoS Med 2005; 2: e45.

5 Handberg A, Levin K, Hojlund K, Beck-Nielsen H. Identification of the oxidized lowdensity lipoprotein scavenger receptor CD36 in plasma: a novel marker of insulin resistance. Circulation 2006; 114: 1169-1176.

6 Handberg A, Norberg M, Stenlund H, Hallmans G, Attermann J, Eriksson JW. Soluble CD36 (sCD36) clusters with markers of insulin resistance, and high sCD36 is associated with increased type 2 diabetes risk. J Clin Endocrinol Metab 2010; 95 1939-1946.

7 Handberg A, Skjelland M, Michelsen AE, Sagen EL, Krohg-Sorensen K, Russell D et al. Soluble CD36 in plasma is increased in patients with symptomatic atherosclerotic carotid plaques and is related to plaque instability. Stroke 2008; 39: 3092-3095.

8 Glintborg D, Hojlund K, Andersen M, Henriksen JE, Beck-Nielsen H, Handberg A. Soluble CD36 and risk markers of insulin resistance and atherosclerosis are elevated in polycystic ovary syndrome and significantly reduced during pioglitazone treatment. Diabetes Care 2008; 31: 328-334.

9 Fernandez-Real JM, Handberg A, Ortega F, Hojlund K, Vendrell J, Ricart W. Circulating soluble CD36 is a novel marker of liver injury in subjects with altered glucose tolerance. J Nutr Biochem 2009; 20: 477-484.

10 Handberg A, Hojlund K, Gastaldelli A, Flyvbjerg A, Dekker JM, Petrie J et al. Plasma sCD36 is associated with markers of atherosclerosis, insulin resistance and fatty liver in a nondiabetic healthy population. J Intern Med 2012; 271: 294-304.

11 Gruarin P, Thorne RF, Dorahy DJ, Burns GF, Sitia R, Alessio M. CD36 is a ditopic glycoprotein with the $\mathrm{N}$-terminal domain implicated in intracellular transport Biochem Biophys Res Commun 2000; 275: 446-454.

12 Alkhatatbeh MJ, Mhaidat NM, Enjeti AK, Lincz LF, Thorne RF. The putative diabetic plasma marker, soluble CD36, is non-cleaved, non-soluble and entirely associated with microparticles. J Thromb Haemost 2011; 9: 844-851.

13 Little KM, Smalley DM, Harthun NL, Ley K. The plasma microparticle proteome. Semin Thromb Hemost 2010; 36: 845-856.

14 Burnier L, Fontana P, Kwak BR, Angelillo-Scherrer A. Cell-derived microparticles in haemostasis and vascular medicine. Thromb Haemost 2009; 101: 439-451.

15 Horstman LL, Ahn YS. Platelet microparticles: a wide-angle perspective. Crit Rev Oncol Hematol 1999; 30: 111-142.

16 Ratajczak J, Wysoczynski M, Hayek F, Janowska-Wieczorek A, Ratajczak MZ. Membrane-derived microvesicles: important and underappreciated mediators of cell-to-cell communication. Leukemia 2006; 20: 1487-1495

17 Enjeti AK, Lincz LF, Seldon M. Microparticles in health and disease. Semin Thromb Hemost 2008; 34: 683-691.

18 Tan KT, Tayebjee MH, Lim HS, Lip GY. Clinically apparent atherosclerotic disease in diabetes is associated with an increase in platelet microparticle levels. Diabet Med 2005; 22: 1657-1662.

19 Mause SF, Weber C. Microparticles: protagonists of a novel communication network for intercellular information exchange. Circ Res. [Research Support, Non-U.S Gov't Review] 2010; 107: 1047-1057.

20 Robert S, Poncelet P, Lacroix R, Arnaud L, Giraudo L, Hauchard A et al. Standardization of platelet-derived microparticle counting using calibrated beads and a Cytomics FC500 routine flow cytometer: a first step towards multicenter studies? J Thromb Haemost 2009; 7: 190-197.

21 Lacroix R, Robert S, Poncelet P, Kasthuri RS, Key NS, Dignat-George F. Standardization of platelet-derived microparticle enumeration by flow cytometry with calibrated beads: results of the International Society on Thrombosis and Haemostasis SSC Collaborative workshop. J Thromb Haemost 2010; 8: 2571-2574.

22 Thorne RF, Meldrum CJ, Harris SJ, Dorahy DJ, Shafren DR, Berndt MC et al. CD36 forms covalently associated dimers and multimers in platelets and transfected COS-7 cells. Biochem Biophys Res Commun 1997; 240: 812-818.

23 Dixon JB, O'Brien PE. Obesity and the white blood cell count: changes with sustained weight loss. Obes Surg 2006; 16: 251-257.

24 Pratley RE, Wilson C, Bogardus C. Relation of the white blood cell count to obesity and insulin resistance: effect of race and gender. Obes Res 1995; 3 : 563-571.

25 Goichot B, Grunebaum L, Desprez D, Vinzio S, Meyer L, Schlienger JL et al. Circulating procoagulant microparticles in obesity. Diabetes Metab 2006; 32: 82-85. 
26 Agouni A, Lagrue-Lak-Hal AH, Ducluzeau PH, Mostefai HA, Draunet-Busson C Leftheriotis $\mathrm{G}$ et al. Endothelial dysfunction caused by circulating microparticles from patients with metabolic syndrome. Am J Pathol 2008; 173: 1210-1219.

27 Leroyer AS, Tedgui A, Boulanger CM. Microparticles and type 2 diabetes. Diabetes Metab 2008; 34(Suppl 1): S27-S32.

28 Tramontano AF, Lyubarova R, Tsiakos J, Palaia T, Deleon JR, Ragolia L. Circulating endothelial microparticles in diabetes mellitus. Mediators Inflamm 2010; 2010: 250476.

29 Omoto S, Nomura S, Shouzu A, Nishikawa M, Fukuhara S, Iwasaka T. Detection of monocyte-derived microparticles in patients with Type II diabetes mellitus. Diabetologia 2002; 45: 550-555.

30 Diamant M, Nieuwland R, Pablo RF, Sturk A, Smit JW, Radder JK. Elevated numbers of tissue-factor exposing microparticles correlate with components of the metabolic syndrome in uncomplicated type 2 diabetes mellitus. Circulation 2002; 106: 2442-2447.

31 Willekens FL, Bosch FH, Roerdinkholder-Stoelwinder B, Groenen-Dopp YA, Werre JM. Quantification of loss of haemoglobin components from the circulating red blood cell in vivo. Eur J Haematol 1997; 58: 246-250.

32 Willekens FLA, Werre JM, Groenen-Döpp YAM, Roerdinkholder-Stoelwinder B, De Pauw B. Bosman GJCGM. Erythrocyte vesiculation: a self-protective mechanism? Br J Haematol 2008; 141: 549-556.

33 Keymel S, Heiss C, Kleinbongard P, Kelm M, Lauer T. Impaired red blood cell deformability in patients with coronary artery disease and diabetes mellitus. Horm Metab Res 2011; 43: 760-765.

34 Bosman GJ, Lasonder E, Groenen-Dopp YA, Willekens FL, Werre JM, Novotny VM. Comparative proteomics of erythrocyte aging in vivo and in vitro. $J$ Proteomics 2010; 73: 396-402.

35 Mallat Z, Benamer H, Hugel B, Benessiano J, Steg PG, Freyssinet JM et al. Elevated levels of shed membrane microparticles with procoagulant potential in the peripheral circulating blood of patients with acute coronary syndromes. Circulation 2000; 101: 841-843.

36 Sabatier F, Darmon P, Hugel B, Combes V, Sanmarco M, Velut J-G et al. Type 1 and type 2 diabetic patients display different patterns of cellular microparticles. Diabetes 2002; 51: 2840-2845.

37 Tsimerman G, Roguin A, Bachar A, Melamed E, Brenner B, Aharon A. Involvement of microparticles in diabetic vascular complications. Thromb Haemost 2011; 106: 310-321.

38 Helal O, Defoort C, Robert S, Marin C, Lesavre N, Lopez-Miranda J et al. Increased levels of microparticles originating from endothelial cells, platelets and erythrocytes in subjects with metabolic syndrome: relationship with oxidative stress. Nutr Metab Cardiovasc Dis 2011; 21: 665-671.
39 Jung KH, Chu K, Lee ST, Bahn JJ, Kim JH, Kim M et al. Risk of macrovascular complications in type 2 diabetes mellitus: endothelial microparticle profiles. Cerebrovasc Dis 2011; 31: 485-493.

40 Esposito K, Ciotola M, Giugliano D. Pioglitazone Reduces Endothelial Microparticles in the Metabolic Syndrome. Arterioscler Thromb Vasc Biol 2006; 26: 1926.

41 Mobarrez F, Egberg N, Antovic J, Bröijersen A, Jörneskog G, Wallén H. Release of endothelial microparticles in vivo during atorvastatin treatment: a randomized double-blind placebo-controlled study. Thromb Res 2012; 129: 95-97.

42 Gonzalez-Quintero VH, Smarkusky LP, Jimenez JJ, Mauro LM, Jy W, Hortsman LL et al. Elevated plasma endothelial microparticles: preeclampsia versus gestational hypertension. Am J Obstet Gynecol 2004; 191: 1418-1424.

43 van lerssel SH, Van Craenenbroeck EM, Conraads VM, Van Tendeloo VF, Vrints $\mathrm{C}$, Jorens PG et al. Flow cytometric detection of endothelial microparticles (EMP): effects of centrifugation and storage alter with the phenotype studied. Thromb Res 2010; 125: 332-339.

44 Chmielewski M, Bragfors-Helin AC, Stenvinkel P, Lindholm B, Anderstam B. Serum soluble CD36, assessed by a novel monoclonal antibody-based sandwich ELISA, predicts cardiovascular mortality in dialysis patients. Clin Chim Acta 2010; 411: 2079-2082.

45 George J, Thoi L, McManus L, Reimann T. Isolation of human platelet membrane microparticles from plasma and serum. Blood 1982; 60: 834-840.

46 Ghosh A, Murugesan G, Chen K, Zhang L, Wang Q, Febbraio M et al. Platelet CD36 surface expression levels affect functional responses to oxidized LDL and are associated with inheritance of specific genetic polymorphisms. Blood 2011; 117: 6355-6366.

47 Love-Gregory L, Sherva R, Schappe T, Qi JS, McCrea J, Klein S et al. Common CD36 SNPs reduce protein expression and may contribute to a protective atherogenic profile. Hum Mol Genet 2011; 20: 193-201.

48 Mandosi E, Fallarino M, Gatti A, Carnovale A, Rossetti M, Lococo E et al. Atorvastatin downregulates monocyte CD36 expression, nuclear NFkappaB and TNFalpha levels in type 2 diabetes. J Atheroscler Thromb 2010; 17: 539-545.

49 Alexander Aguilera A, Hernández Díaz G, Lara Barcelata M, Angulo Guerrero O, Oliart Ros RM. Induction of $\mathrm{Cd} 36$ expression elicited by fish oil PUFA in spontaneously hypertensive rats. J Nutr Biochemistry 2006; 17: 760-765.

50 Griffin E, Re A, Hamel N, Fu C, Bush H, McCaffrey T et al. A link between diabetes and atherosclerosis: glucose regulates expression of CD36 at the level of translation. Nat Med 2001; 7: 840-846.

(c) (9) (2) This work is licensed under a Creative Commons Attribution-
NonCommercial-ShareAlike 3.0 Unported License. To view a copy of this license, visit http://creativecommons.org/licenses/by-nc-sa/3.0/

Supplementary Information accompanies the paper on the Nutrition and Diabetes website (http://www.nature.com/nutd) 